\title{
Emergency Department Treatment of Cyclic Vomiting Syndrome
}

\author{
Robert M Issenman* \\ Professor of Pediatrics, McMaster Children's Hospital, Canada
}

*Corresponding author: Robert M Issenman, Professor of Pediatrics, McMaster Children's Hospital, Hamilton, Ontario, Canada.

Received Date: September 28, 2020

Published Date: October 15, 2020

\section{Guest Editorial}

A recent medical article describes a patient's frustration dealing with her local Emergency Department Sickle Cell Disease painful crisis. The patient declares "My relationship with the emergency room is that there is no relationship" [1]. It is not unusual for a chronic disease patient to become frustrated by their inability to obtain predictable Emergency Department Care. As a physician caring for Cyclic Vomiting Syndrome patients, I thought it would be relatively simple to provide patients with, a letter identifying the best form of treatment for their condition. During follow-up clinic visits, I was surprised to learn that these letters were often ignored. In some cases, these letters were thrust back at the patient's family with a gesture that declared "We're not doing that". A survey of US patients using Emergency Department confirmed that directive letters are ignored in approximately $50 \%$ of visits [2].

Having been given the opportunity to lead the Emergency Department at our children's hospital provided some insight into the challenge. As acting Department Chief for two years, I learned that there was some rational to the resistance of Emergency Department physicians being "told what to do" by "outside" doctors. The first is that hospital physicians are usually engaged as independent contractors rather than hospital employees. Physicians are held independently accountable for their care. They are uniquely vulnerable to being blamed (or sued) for patient misadventures that are often a problem with the medical system, not their care. Like police, physicians are taught to bring a certain skepticism to "received wisdom". The defense that "I based my actions on the suggestion of another physician" is not particularly strong. They are sensitive to being considered "handmaidens" of outside physicians who send patients down to the Emergency Department with unrealistic expectations of having an x-ray, ultrasound, MRI or even hospital admission. In most instances, they are seeing patients they don't know and likely won't see again. Their concern about being manipulated by "drug seeking" patients is ever more real with the appreciation of the abuses of the Opioid Epidemic.

Nevertheless, there are small groups of patients who despite ongoing medical care need to come to the Emergency Department for medical treatment. In the pediatric age group, Complex Migraine, Cyclic Vomiting Syndrome, Sickle Cell Crisis, and Inborn Errors of Metabolism come to mind. Even though these events are not life-threatening, the principle of the "golden hour" developed in the treatment of chest pain, stroke and diabetes should be applied to these groups. Early treatment results in milder illness, shorter duration of stay and fewer hospitalizations [3]. It is better for both the patient and the Emergency Department that these patients be rapidly identified and the treatment that has worked best in the past be commenced quickly. Once we started to think of the problem as a process problem rather than as a physician problem, solutions became more obvious. Working with the Emergency Department administration (a series of Head Nurses), we developed an Alert System issuing the patient with an "Alert" card that identified the existence of the patient's Clinical Treatment Plan in the Hospital's information system. There was more success after an Emergency 
Department registration clerk suggested treatment plan be placed on the patient's chart at the registration desk as a means of providing a consistent process for every visit.

As a result of this experience, we would suggest the following if you are a patient struggling to get predictable care for a chronic disease from your local Emergency Department.

1. Identify a physician advocate to help develop your treatment plan. It is best if this physician has established a "relationship of trust" with the emergency department staff.

2. The physician advocate should ideally try to contact the Emergency Department Medical Chief to get agreement on the plan to be used during ED visits.

3. In other instances, the physician advocate or the patient themselves should seek to meet with the Emergency Department Head Nurse at some time between crisis events, to negotiate the treatment plan.

4. In the event of encountering resistance, it might be reasonable to contact the hospital's "Patient Experience" department to get a dialogue going. These departments are set up to deal with complaints and might welcome the opportunity to meet with a patient to avoid a complaint.
5. In the absence of open-minded response from the Emergency Department management, the hospital might be convinced to open a dialogue with direction from the Hospital Board.

Lastly, don't expect an overnight solution. The process described above represented a 10-year journey in a willing institution. However, the adaptation of the medical system to Covid has taught us that, if needs be, medical systems can adapt much more quickly than previously thought.

\section{Acknowledgement}

None.

\section{Conflict of Interest}

No conflict of interest.

\section{References}

1. Kevin J Solverson, Christopher J Doig (2020) A fatal case of pneumococcal sepsis years after splenectomy. CMAJ 192(40): E800-E802.

2. Thangam Venkatesan, Sally Tarbell, Kathleen Adams, Jennifer McKanry, Trish Barribeau, et al. (2010) A survey of Emergency Department Use in patients with cyclic vomiting syndrome. BMC Emerg Med 10: 4 .

3. Jeffrey L Saver, Eric E Smith, Gregg C Fonarow, Mathew J Reeves, Xin Zhao, et al. (2010) The "golden hour" and acute brain ischemia: presenting features and lytic therapy in $>30,000$ patients arriving within 60 minutes of stroke onset. Stroke 41: 1431-1439. 